\title{
Circumumbilical incision for mobile unilocular abdominal cysts
}

\author{
Mohammad Ahmad Al Ekrashy, MD
}

Department of Pediatric Surgery, Zagazig University, Egypt.

\begin{abstract}
Aim: The aim of this study was to evaluate the accessibility of circumumbilical incision for excision of mobile unilocular abdominal cysts and its cosmetic results as compared with other traditional incision in pediatric abdominal surgery.

Patients and methods: Twenty patients with mobile unilocular abdominal cysts of different origin were prospectively selected for periumbilical incision for excision of cysts. Preoperative assessment of the cyst was done either by ultrasonography or CT for assuring of being mobile unilocular cysts and being solitary or multiple. Postoperative assessment of the cosmetic results, length of hospital stay and return to normal activity were done and were significantly different between periumbilical incision and traditional laparotomy incisions.

Results: Periumbilical incision is safe and provides accepted accessibility for mobile unilocular abdominal cysts excision and reduces postoperative wound complications and hospital stay with better cosmetic results.
\end{abstract}

\section{Introduction:}

Many abdominal exploratory incisions can be done to excise mobile abdominal cysts according to its position and relations. In infants and young children supraumbilical transverse incision is preferred as a good exploratory incision for the most of the abdomen. But in mobile pelvic masses like ovarian cysts the suprapubic Pfannensteil incision is the suitable one, but there're many medicoleagal and social restrictions for that incision for virgin females so, if the periumbilical incision can be suitable for managing these cases it's considered a magical solution for these cases.

Understanding the detailed anatomy of the umbilical and periumbilical region is important to assume best exploratory and cosmetic results. Embryologically, the umbilicus is a midline fusion of the medial aponeurotic borders of both rectus abdominis aponeuroses around the umbilical cord. This fusion may take place around the 10th week, after the herniated midgut returns to the peritoneal cavity.

Remember the following four anatomic entities, which pass through the umbilical ring in the newly born child:

Left umbilical vein (round ligament of the liver)

Urachus (median umbilical ligament)

Two umbilical arteries (medial umbilical ligaments).

The umbilicus is located at the center of the umbilical region. For all practical purposes, the umbilicus is a scar. It is not the same in all individuals. Its boundaries are the epigastric area above, the hypogastric area below, and the right and left lumbar areas laterally.

The umbilicus is essentially at the vertical midpoint of the linea alba. It marks the junction of the lower end of the well-formed upper linea alba and the beginning of the poorly-defined lower linea alba (Skandalakis et al 2004).

Umbilical Ring:

The medial umbilical ligaments (obliterated umbilical arteries) and the urachus (obliterated allantoic duct) participate in the formation of the fibrous umbilical ring. The round ligament (obliterated umbilical vein) arises from the inferior margin of the ring and passes superiorly in the falciform ligament. 
Umbilical fascia:

- In 36\%, a localized thickening of the transversalis fascia in this area, named the umbilical fascia, covers the umbilical ring in toto. This fascial "buffer" can protect against the genesis of an umbilical hernia.

- In 38\% of individuals, the umbilical fascia covers only the upper part of the umbilical ring.

- In $6 \%$ of individuals, the umbilical fascia covers only the lower part of the umbilical ring.

- In $4 \%$ of individuals, the umbilical fascia is located above the ring.

- In $16 \%$ of individuals, the umbilical fascia is absent. ${ }^{1}$

\section{Patients and methods:}

This study was carried out in our pediatric surgery unit. It was carried on twenty patients; 12 ovarian cysts, 4 of them were complicated, 4 mobile mesenteric pedunculated cysts and 4 urachal cysts. 16 patients were females and 4 males. The ages were ranging between two months and four years, with average age 17.2 months. Cysts were removed through circuminfra-umbilical approach. Early postoperative events and complications were registered. After discharge from hospital regular follow up in outpatient clinic and delayed post operative complications were also registered. Only one case of seroma was encountered after excision of a complicated right ovarian cyst, which was managed by evacuation then compressing dressing was done over the umbilical region and cefotriaxone injection.

Another case with angular incisional hernia was encountered after excision of another simple ovarian cyst, this hernia was managed conservatively and disappeared after 6 months.

Surgical technique; under general anaethesia and endo tracheal intubation, a nearly half sphere infraumbilical incision through skin and subcutaneous tissue, better by scalpel No 11 while skin at the expected two ends of the incision are elevated by two toothed forceps. Then the umbilical scar is freed of its fascia and the rectus sheath is incised at both sides till a limit that the incised skin allows. Insert two or more Langenbeck's retractors to allow visualization of the abdominal cyst. The cyst is fixed between the retractors and the cyst fluidy content is aspirated by suitable sized syringe and needle and the needle is removed while an artery forceps grasps and blocks the puncture site to prevent intra peritoneal leakage of the fluid. Now the artery forceps can extract the lax cyst extra abdominally easily and excised.

The abdominal wall is closed in mass by continuous vicryl $2 / 0$ or $3 / 0$ according to age. The umbilical scar is fixed to the sheath using $4 / 0$ vicryl stitch. 3 to 4 subcutaneous inverted sutures; the skin is closed in subcuticular sutures. Compressing dressing was applied over the umbilicus for 4 to 5 days.

Various types of cysts were excised through this incision; mesenteric pedunculated cyst, right ovarian cyst and urachal cysts.

\section{Results:}

The study was carried on 20 patients with three different causes of abdominal cysts, Table (1).

Postoperative hospital stay was around 24 hours for all cases, through this time the patients started oral feeding and was discharged to outpatient clinic.

Postoperative complications were arranged in Table (2).

The seroma was evacuated and compressive dressing was applied and parentral antibiotic resolved the condition.

The incisional hernia was angular and was managed conservatively and disappeared after around six months.

Wound appearance was very satisfactory to parents as the wound and umbilicus appeared normal.

\section{Discussion:}

Using circumumbilical approach for pyloromyotomy ${ }^{4}$ has lead to a realization of its potential for the management of many routine abdominal operative procedures in the neonates as well as oncologic surgery.

It was used for malrotation, intestinal atresia, NEC, spontaneous perforations, meconium ileus and intestinal duplication. ${ }^{3}$ 


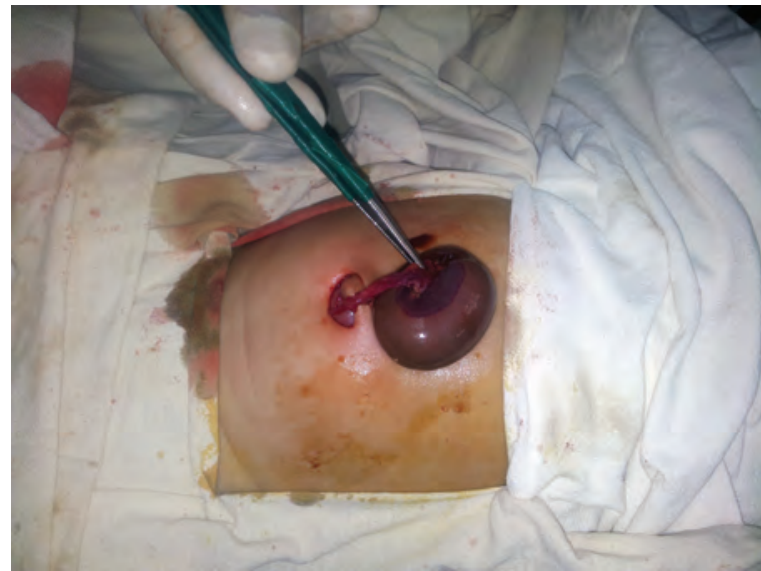

Figure (1): Simple pedunculated mesenteric cyst excision through the umbilical incision.

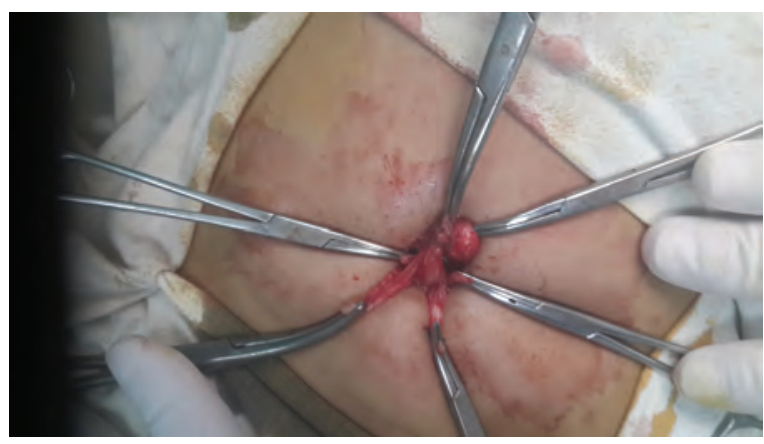

Figure (3): Excision of urachal cyst through circumumbilical approach.

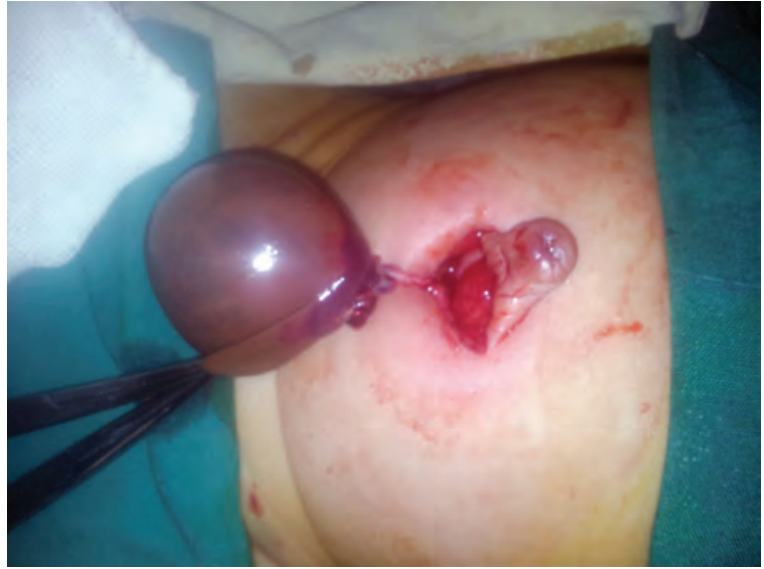

Figure (2): Complicated right ovarian cyst extracted through infraumbilical incision.

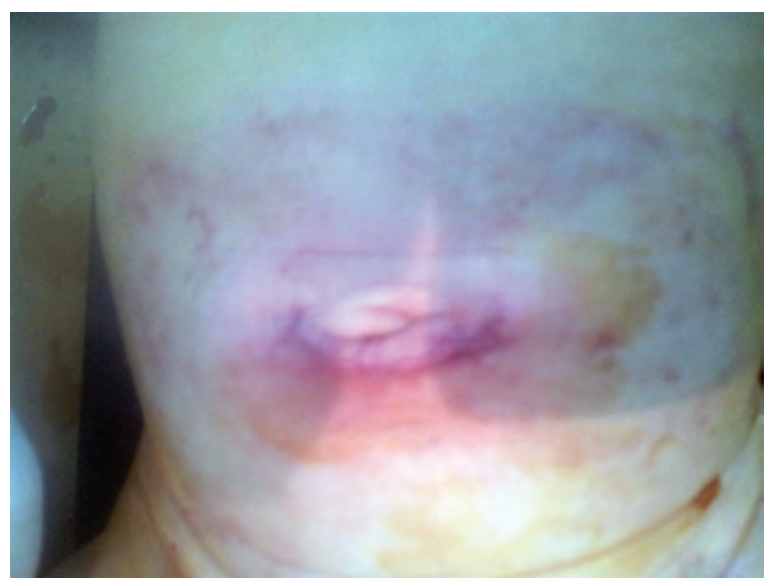

Figure (4): Circum-infraumbilical incision after closure in subcuticular stitches.

Table (1):

\begin{tabular}{|l|l|}
\hline Number of cases & \multicolumn{1}{|c|}{ Type of cyst } \\
\hline 16 & Ovarian cyst \\
\hline 4 & Mesenteric cyst \\
\hline 4 & Urachal cyst \\
\hline
\end{tabular}

Table (2):

\begin{tabular}{|l|l|}
\hline Postoperative complications & Number of cases \\
\hline Wound seroma & One \\
\hline Incisional hernia & One \\
\hline
\end{tabular}

It has the advantage of safe exposure of the abdominal contents and the ability to deliver bowel through the wound and all procedures are done under full vision.

Fiona et al 2009, ${ }^{3}$ used muscle sparing procedure which doesn't require the creation of a subcutaneous pocket that's prone to seroma and infection and wound drainage was not necessary.

We have used muscle cutting technique transversely after creating a subcutaneous space to give a wider exposure and a non limited exploratory view to most of the abdominal contents as the incised skin is freely 
mobile in all directions using appropriate abdominal retractors.

Good closure of the abdominal wall and good compressive dressing after subcuticular closure of skin have prevented seroma, infection and incisional hernia in all cases except one case of seroma, and one case of angular incisional hernia.

Seroma was managed by evacuation compression and parentral antibiotics and the angular incisional hernia resolved spontaneously after around six months.

Postoperative complications are comparable with conventional approaches for similar operations. ${ }^{3}$

We have closed the wound in circumferential manner leaving small skin scar without interruption of the abdominal wall. The scar is well camouflaged within the peri umbilical skin and is much appreciated by parents.

There is an appreciable reduction in postoperative pain and enhanced recovery. ${ }^{3}$

Fiona et al $2009^{3}$ had two incisional hernias which can be avoided by particular attention that there's no tension or strangulation of tissues by the sutures. The circumsupraumbilical approach is an alternative to laparoscopy combining safety and minimal disruptive surgery. It requires no additional equipments or particular skills and leads to an aesthetic scar. ${ }^{3}$

Operative time didn't constitute an issue in our study.

The circumumbilical incision if it was found to be inappropriate for the procedure there are two options to solve this problem; the first is to extend the wound laterally to give an omega shaped incision, the second is to add another incision to fulfill the procedure like Pfannensteil incision in lower abdominal conditions and transverse supra umbilical incision in upper abdominal conditions.

Aspiration of the fluid content of the cyst made us dispense extending the incision into Omega shape also there was no need for combination of another aesthetic incision.

In a study for Tajiri et al ${ }^{6} 14$ neonates with surgical diseases (3 hypertrophic pyloric stenoses, 3 ileal atresias, 2 jejunal atresias,
1 duodenal stenosis, 1 duodenal atresia, 2 ovarian cysts, 1 malrotation, and 1 segmental dilatation of ileum), were treated using a transumbilical approach by means of a half circumumbilical incision. In 10 of 14 cases, the umbilicus was incised on its upper half circumference, while the umbilicus of 4 cases was incised on its lower half circumference. In one ileal atresia patient with a remarkable degree of oral intestinal dilatation, a slight additional transverse incision was added. In four cases ( 1 case with ileal atresia, 2 cases of an ovarian cyst, and 1 case with a segmental dilatation of the ileum), laparoscopy-assisted transumbilical surgery was performed. In all cases, no operative complications were encountered. Postoperatively, there was no wound in appearance and the umbilicus appeared to be normal.

The length of hospital stay was 24 hours through that time the patient had started oral feeding and then he was discharged home for follow up in out patient clinic after three days for dressing.

In a study for Megha and Jacob 2011, ${ }^{2}$ One hundred thirty-two patients underwent a laparotomy through a transverse abdominal incision $(\mathrm{n}=106)$ or a circumumbilical incision $(\mathrm{n}=26)$. Baseline characteristics between groups were similar. No differences were found when comparing operative time, postoperative days on a ventilator, narcotic infusion, time to full feeds, length of hospital stay, incidence of surgical site infection, and bowel obstruction. Although more incisional hernias occurred in the circumumbilical incision group (38\%) than the transverse abdominal incision group (6\%), all hernias in the circumumbilical group resolved without intervention, whereas 33\% required surgical repair in the transverse abdominal group.

In another series for Murphy et al, ${ }^{5}$ a total of 55neonates with a gestational age ranging from 28 to 42 weeks had 57 operative procedures. The indications were: nonrotation of midgut in 18; intestinal atresia in 18; necrotizing enterocolitis/spontaneous perforation in 10; meconium ileus in 5; intestinal duplication in 2; patent vitellointestinal duct (VID) in 2. No conversion to a standard transverse 
incision was necessary in any case. However, an omega extension was made in four patients. The complications encountered included wound infection in one; caecal perforation in one and incisional hernia in two cases. Subsequent follow-up revealed that all incisions had healed and the scars were almost imperceptible as affirmed by parental satisfaction during outpatient clinic consultation.

\section{Conclusion:}

The circumumbilical approach is a safe, flexible and easily reproducible approach providing adequate exposure for most abdominal surgeries in the neonate resection of unilocular mobile abdominal cysts and is done very easily leaving no gross morbidity nor any ugly scars. This incision decreases the time of hospital stay, decreases post operative pain with early postoperative recovery and less wound complications. The low complication rate and good aesthetic outcome which are much appreciated by parents make the approach deserving trial before doing another traditional incision.

\section{Reference}

1- Skandalakis J, Colborn GL, Weidman TA, Foster RS, Kingsnorth AN, Skandalakis LJ, Mirilas PS: Abdominal wall and hernia. McGrow Hill' access surgery, 2004; Chapter 9.

2- Suri M, Langer JC: A comparison of circumumbilical and transverse abdominal incisions for neonatal abdominal surgery. Journal of Pediatric Surgery 2011; 46(6): 1076-1080.

3- Murphy FJ, Mohee A, Khalil B, Lall A, Morabito A, Bianchi A: Versatility of the circumumbilical incision in neonatal surgery. Pediatric Surgery International 2009; 25(2): 145-147.

4- Ali Gharaibeh KI, Ammari F, Qasaimeh G, Kasawneh B, Sheyyab M, Rawashdeh M: Pyloromyotomy through circumumbilical incision. J R Coll Surg Edinb 1992; 37(3): 175-176.

5- Murphy FJ, Mohee A, Khalil B, Lall A, Morabito A, Bianchi A: Versatility of the circumumbilical incision in neonatal surgery. Pediatr Surg Int 2009; 25(2): 145-147.

6- Tajiri T, Ieiri S, Kinoshita Y, Masumoto K, Nishimoto Y, Taguchi T: Transumbilical approach for neonatal surgical diseases: Woundless operation. Pediatr Surg Int 2008; 24(10): 1123-1126. 